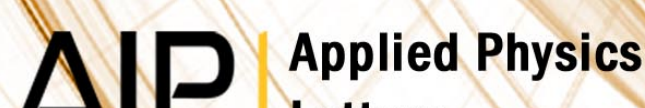 \\ Letters
}

\section{Imaging dielectric relaxation in nanostructured polymers by frequency modulation electrostatic force microscopy}

C. Riedel, R. Sweeney, N. E. Israeloff, R. Arinero, G. A. Schwartz et al.

Citation: Appl. Phys. Lett. 96, 213110 (2010); doi: 10.1063/1.3431288

View online: http://dx.doi.org/10.1063/1.3431288

View Table of Contents: http://apl.aip.org/resource/1/APPLAB/v96/i21

Published by the American Institute of Physics.

\section{Related Articles}

Electron paramagnetic resonance investigation of polar nanoregions mobility in the relaxor PbMg1/3Nb2/3O3 and solid solutions $\mathrm{PbMg} 1 / 3 \mathrm{Nb} 2 / 3 \mathrm{O} 3-\mathrm{PbTiO} 3$

\section{J. Appl. Phys. 111, 014104 (2012)}

The dielectric relaxation behavior of $(\mathrm{Na} 0.82 \mathrm{~K} 0.18) 0.5 \mathrm{Bi} 0.5 \mathrm{TiO} 3$ ferroelectric thin film

J. Appl. Phys. 110, 124109 (2011)

Dielectric and spin relaxation behaviour in $\mathrm{DyFeO} 3$ nanocrystals

J. Appl. Phys. 110, 124301 (2011)

Dielectric relaxation and alternating current conductivity of polyvinylidene fluoride doped with lanthanum chloride J. Appl. Phys. 110, 114119 (2011)

Defects control for improved electrical properties in (Ba0.8Sr0.2)(Zr0.2Ti0.8)O3 films by Co acceptor doping Appl. Phys. Lett. 99, 232910 (2011)

\section{Additional information on Appl. Phys. Lett.}

Journal Homepage: http://apl.aip.org/

Journal Information: http://apl.aip.org/about/about_the_journal

Top downloads: http://apl.aip.org/features/most_downloaded

Information for Authors: http://apl.aip.org/authors

\section{ADVERTISEMENT}

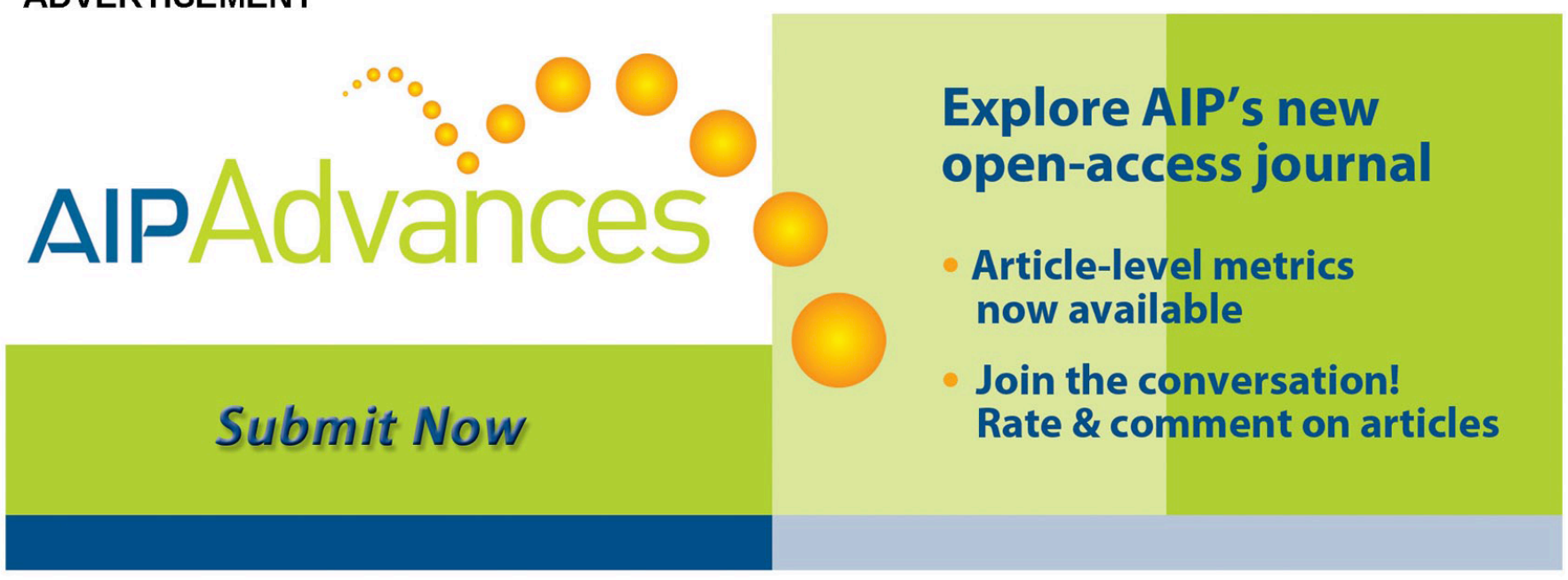




\title{
Imaging dielectric relaxation in nanostructured polymers by frequency modulation electrostatic force microscopy
}

\author{
C. Riedel,,${ }^{1,2, a)}$ R. Sweeney ${ }^{3}$ N. E. Israeloff, ${ }^{3}$ R. Arinero, ${ }^{1}$ G. A. Schwartz, ${ }^{4}$ A. Alegria, ${ }^{2}$ \\ Ph. Tordjeman, ${ }^{5}$ and J. Colmenero ${ }^{2,4}$ \\ ${ }^{1}$ Institut d'Electronique du Sud (IES), UMR CNRS 5214, Université Montpellier II, CC 082, \\ Place E. Bataillon, 34095 Montpellier Cedex, France \\ ${ }^{2}$ Departamento de Física de Materiales, Facultad de Química, UPV/EHU, Apartado 1072, \\ 20080 San Sebastián, Spain and Donostia International Physics Center, Paseo Manuel de Lardizabal 4, \\ 20018 San Sebastián, Spain \\ ${ }^{3}$ Department of Physics, Northeastern University, Boston, Massachusetts 02115, USA \\ ${ }^{4}$ Centro de Física de Materiales CSIC-UPV/EHU, Edificio Korta, 20018 San Sebastián, Spain \\ ${ }^{5}$ Institut de Mécanique des Fluides (IMFT), Université de Toulouse-CNRS, 1 Allée du Professeur Camille \\ Soula, 31400 Toulouse, France
}

(Received 18 February 2010; accepted 27 April 2010; published online 25 May 2010)

\begin{abstract}
We have developed a method for imaging the temperature-frequency dependence of the dynamics of nanostructured polymer films with spatial resolution. This method provides images with dielectric compositional contrast well decoupled from topography. Using frequency-modulation electrostatic-force-microscopy, we probe the local frequency-dependent (0.1$100 \mathrm{~Hz}$ ) dielectric response through measurement of the amplitude and phase of the force gradient in response to an oscillating applied electric field. When the phase is imaged at fixed frequency, it reveals the spatial variation in dielectric losses, i.e., the spatial variation in molecular/dipolar dynamics, with $40 \mathrm{~nm}$ lateral resolution. This is demonstrated by using as a model system; a phase separated polystyrene/polyvinyl-acetate (PVAc) blend. We show that nanoscale dynamic domains of PVAc are clearly identifiable in phase images as those which light-up in a band of temperature, reflecting the variations in the molecular/dipolar dynamics approaching the glass transition temperature of PVAc. (C) 2010 American Institute of Physics. [doi:10.1063/1.3431288]
\end{abstract}

Broadband dielectric spectroscopy (BDS) is a well established technique that enables measurement of molecular dynamics of bulk materials with permanent molecular dipoles. Recent advances have made it possible to probe the dynamics of thin polymer films with a thicknesses as low as $5 \mathrm{~nm}$, enabling the study of interfaces. ${ }^{1,2}$ However, BDS has poor spatial resolution and the interpretation of the results from nanostructured polymers is always model dependent. ${ }^{3}$ Concerning local dielectric characterization, on the one hand, various atomic force microscopy based methods have been developed to image the dc dielectric constant $\varepsilon$ at nanoscale. Fumagalli et al. ${ }^{4}$ have developed the so-called "nanoscale capacitance microscopy," where the microscope is equipped with a subattofarad low-frequency capacitance detector. The same group also proposed a method based on the detection of the dc electrostatic force to image the dielectric constant of a purple membrane patch. ${ }^{5}$ By measuring the dc electrostatic force gradient, we have been able to obtain a quantitative value of $\varepsilon$ in one point ${ }^{6}$ and a map of the dielectric constant of a nanostructured polymer film. ${ }^{7}$ On the other hand, in previous work we have shown that the frequency response of the real and imaginary parts of the dielectric constant $\left[\varepsilon^{*}(\omega)=\varepsilon^{\prime}-i \varepsilon^{\prime \prime}\right]$ can be investigated using frequency modulation electrostatic force microscopy (FM-EFM) ${ }^{8,9}$ Measurements made in one spatial point gives direct information about the local dynamics of the sample area probed by the tip sample interaction. In the following, we will detail how we have been able to combine imaging

\footnotetext{
a) Author to whom correspondence should be addressed. Electronic mail: riedel@ies.univ-montp2.fr.
}

and measurement of the frequency dependence of the dielectric response to map the dipolar/molecular dynamics of a nanostructured thin film of a phase separated polymer blend.

In order to maximize contrast, we have chosen to work with polystyrene (PS- $\left[\mathrm{C}_{8} \mathrm{H}_{8}\right]_{\mathrm{n}}-\mathrm{M}_{\mathrm{w}}=70950 \mathrm{~g} / \mathrm{mol}$ ) and poly(vinyl-acetate) $\left(P V A c-\left[\mathrm{C}_{4} \mathrm{H}_{6} \mathrm{O}_{2}\right]_{\mathrm{n}}-\mathrm{M}_{\mathrm{w}}=33200 \mathrm{~g} / \mathrm{mol}\right)$ for which the bulk dielectric response has been previously well characterized. ${ }^{10-12}$ A solution containing 25\% PVAc and $75 \%$ PS (by mass) and having a total concentration of $1 \%$ polymer in toluene has been spin-coated over a conductive film $(\mathrm{Au})$ on a mica substrate. The samples are dried at $70{ }^{\circ} \mathrm{C}$ for $1 \mathrm{~h}$ under ambient conditions and annealed at $35{ }^{\circ} \mathrm{C}$ overnight in vacuum to remove any trace of solvent. The resulting polymer films consist of a $25 \mathrm{~nm}$ thick matrix with island-like features having an average diameter of about $300 \mathrm{~nm}$ and a maximum height of about $25 \mathrm{~nm}$ above the reference level of the matrix (Fig. 1). In the investigated range of frequency $(0.1-100 \mathrm{~Hz})$, we expect to detect the so-called dielectric alpha-relaxation of PVAc (correlated with segmental motion ${ }^{12}$ ) slightly above its glass transition temperature; $\mathrm{T}_{\mathrm{g}} \sim 35^{\circ} \mathrm{C}$. Therefore we have explored the temperature range between 25 and $50{ }^{\circ} \mathrm{C}$ in order to follow the evolution of this dynamic process. The $\mathrm{T}_{\mathrm{g}}$ of PS being $\sim 105^{\circ} \mathrm{C}$, no measurable relaxation is expected in our range of temperature and frequency. These two polymers are immiscible, even at the nanoscale and form phase separated domains.

Experiments were carried out by FM-EFM under ultrahigh vacuum (UHV) with a variable temperature stage (RHK UHV 350). The temperature was measured with a small thermocouple clamped to the sample surface. 


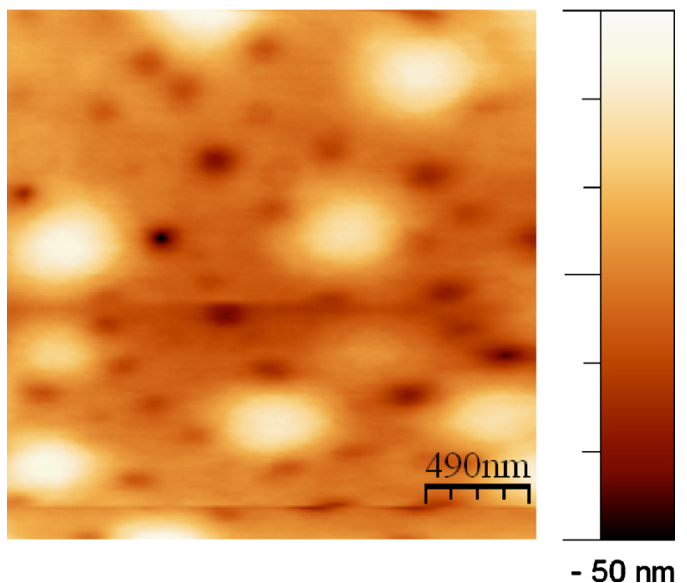

FIG. 1. (Color online) Topography of the thin PS/PVAc polymer film. The thickness of the matrix is approximately $25 \mathrm{~nm}$, whereas islands rise to $50 \mathrm{~nm}$ from the substrate.

Nanosensors ATEC EFM cantilevers with 4:1 aspect ratio and $25 \mathrm{~nm}$ tip radius, spring-constant $\mathrm{k}=2 \mathrm{~N} / \mathrm{m}$, are used so that $\mathrm{V}_{\mathrm{dc}}$ and $\mathrm{V}_{\mathrm{ac}}$ bias voltages can be applied to the tip. In FM-EFM, the cantilever is oscillated at its resonance frequency, $\mathrm{f}_{0}=70 \mathrm{kHz}$, and the resonance frequency shift, $\delta \mathrm{f}$, due to tip-sample interaction forces, is detected with very high resolution using a Nanosurf Easy-PLL Phase-LockedLoop detector. This parameter is used as a feedback for controlling the tip-sample distance $\mathrm{z}$ and allows us to obtain the topography as shown in Fig. 1. Additional signals are those measured as a result of the electrodynamic interaction between the conducting tip and the polymer sample. As the force gradient $\mathrm{dF} / \mathrm{dz}$ is proportional to the square of the voltage, a sinusoidal voltage $\mathrm{V}_{\mathrm{ac}}=\mathrm{V}_{0} \sin (\omega t)$ produces a $2 \omega$ component. This component can be detected as a modulation in the $\delta \mathrm{f}$ signal using a lock-in amplifier. The obtained $\mathrm{V}_{2 \omega}$ signal is related to $\mathrm{dF} / \mathrm{dz}$ by the following: $\mathrm{V}_{2 \omega}=(\mathrm{A} / 4 \mathrm{k})$ $\times(\mathrm{df} / \mathrm{dz}) \mathrm{f}_{0}$, where $\mathrm{A}$ is the instrument gain. ${ }^{8}$ As the force gradient is a function of the dielectric permittivity, ${ }^{6-9}$ any measurable dielectric losses in the material will be detected as a phase shift in the $\mathrm{V}_{2 \omega}$ signal.

Two different experiments were performed to study successively the thin polymer blend. The first is similar to that describe in Refs. 8 and 9 and consisted in recording the phase in one point at different frequencies and constant temperatures. During the acquisition of the dielectric spectrum, the feedback of the PLL detector (typically $\delta \mathrm{f}_{0}=15 \mathrm{~Hz}$ ) was turned off in order to avoid any coupling with the corresponding electrical frequency. We apply a $\mathrm{V}_{\mathrm{ac}}=\mathrm{V}_{0} \sin (\omega t)$ voltage at different frequencies $(\omega / 2 \pi)$ varying from 0.1 to $120 \mathrm{~Hz}$. The frequency range is limited at low frequencies only by the time of acquisition and at high frequencies by the bandwidth of the FM electronics. A preliminary step consists of measuring the reference phase of $\mathrm{V}_{2 \omega}, \Phi_{\text {ref }}$, at $19^{\circ} \mathrm{C}$ where both polymers are in the glassy state and have no measurable dielectric relaxation. The difference between this reference phase and the phase measured in the experiments, $\Delta \Phi=\Phi_{\text {ref }}-\Phi$, is used to characterize the dielectric dissipation. During the relaxation, due to the loss of energy to align the molecular dipoles in the field, a peak is observed in $\Delta \Phi(\omega)$. Using BDS experiments on bulk PVAc, the macroscopic phase of the dielectric permittivity, invtan $\left(\varepsilon^{\prime \prime} / \varepsilon^{\prime}\right)$,

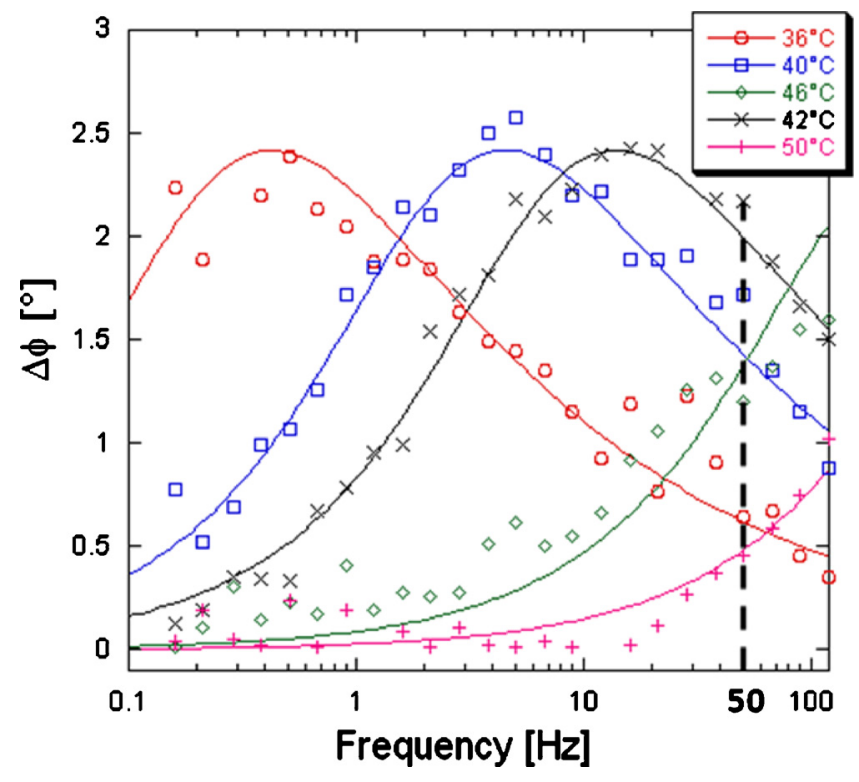

FIG. 2. (Color online) Phase shift measured as a function of the frequency over an island of PVAc. Statistical error is 0.25 for $\mathrm{f}>1 \mathrm{~Hz}$ and 0.35 for $\mathrm{f} \leq 1 \mathrm{~Hz}$. Lines are fits derived from a KWW model with amplitude and shape parameters fixed for all curves. The dashed line indicates the imaging frequency of Fig. 3.

peaks at about $16^{\circ}$ for frequencies of $0.1-100 \mathrm{~Hz}$, similar to what was found for $\Delta \Phi(\omega)$ of thick films $(1 \mu \mathrm{m})$ of PVAc using the present FM-EFM technique. ${ }^{8}$ For thinner films of PVAc, we found that the peak in $\Delta \Phi(\omega)$ is considerably reduced, to $\sim 3^{\circ}$ for $50 \mathrm{~nm}$ films. This can be understood by recognizing that the force gradient is a complex function of the dielectric permittivity, film thickness, tip radius, and tip height that requires numerical modeling. ${ }^{7}$ Whereas the macroscopic phase is proportional to fractional increase in permittivity, $\Delta \varepsilon / \varepsilon$, upon warming through the glass transition, the $\Delta \Phi(\omega)$ we measure here is proportional to the fractional increase in force gradient, $\Delta(\mathrm{dF} / \mathrm{dz}) /(\mathrm{dF} / \mathrm{dz})$. This fraction is found in simulations to be reduced with reduced film thickness due to a relatively larger constant background $\mathrm{dF} / \mathrm{dz}$.

Figure 2 shows $\Delta \Phi(\omega)$ over an island at different temperatures. The fitting lines were obtained using a Kohlrausch-Williams-Watts response function, $\exp (-\mathrm{t} / \tau)^{\beta},{ }^{13,14}$ as a convenient way to describe the experimental result. Maximum value of $\Delta \Phi$ and shape parameter $(\beta=0.42)$ were fixed by fitting the data measured at $40{ }^{\circ} \mathrm{C}$. Keeping these parameters constant and using only a free timescale parameter we obtained a rather satisfactory description of the data, i.e., the shape of the peak does not depend on temperature within the uncertainties. The peak frequencies and shape of these peaks are nearly identical to those found in pure PVAc thick films. Measurements at the same temperatures have been performed over the matrix and the response (not shown) is always flat $\left(\Delta \Phi<0.1^{\circ}\right)$. Therefore, the frequency spectrum permits us to characterize locally the dynamical properties of the sample, which shows that the matrix is composed of PS whereas islands are dynamic domains of PVAc, in agreement with Ref. 7 which imaged static dielectric response.

The second experiment involved scanning the sample surface while a $50 \mathrm{~Hz} \mathrm{~V}_{\mathrm{ac}}$ is applied to the tip. In order to keep a constant tip-sample distance, the feedback of the PLL 

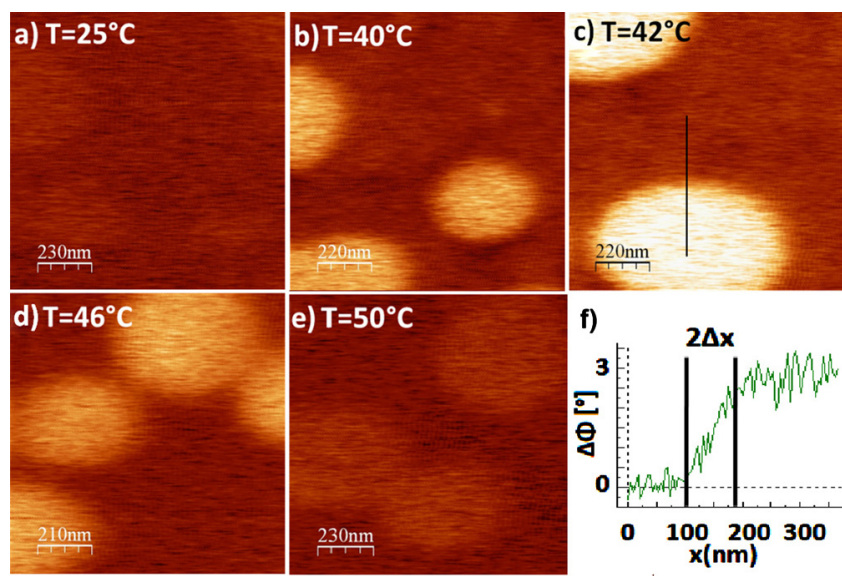

FIG. 3. (Color online) Image of the phase shift recorded at $50 \mathrm{~Hz}$ on a PS/PVAc film for various temperatures. Brighter areas correspond to higher losses, indicative of segmental relaxation on $\sim 3 \mathrm{~ms}$ time scales in PVAc. The profile recorded on the black line at $42{ }^{\circ} \mathrm{C}$ is shown in (f). The phase jump at the PS/PVAc boundary gives an estimated lateral resolution, $\Delta \mathrm{x}$ $\sim 40 \mathrm{~nm}$

is used during the scan. Figure 3 presents images of $\Delta \Phi$ recorded at different temperatures. All images have been offset to the same phase-shift color scale using WSXM. ${ }^{15}$ The phase recorded on the flat matrix areas is always near zero, as expected for PS. On the other hand all values measured on the PVAc islands are, within experimental errors, the same as those measured using the frequency sweep in one single point at $50 \mathrm{~Hz}$ (Fig. 2). At $25^{\circ} \mathrm{C}$, the image is homogeneous; there is no dielectric relaxation occurring in the islands of PVAc. This demonstrates that this method is only sensitive to dipolar relaxation and not topographical effects. When the temperature is increased to $36{ }^{\circ} \mathrm{C}$ we begin to see strong contrast in the images as the high frequency wing of the dielectric loss peak starts to contribute at $50 \mathrm{~Hz}$ in the PVAc regions. The maximum contrast is found at $42{ }^{\circ} \mathrm{C}$. Further increasing the temperature reduces the contrast as the loss peak shifts to higher frequencies and only the lower frequency contributions are detected. As the shape of the relaxation does not depend significantly on temperature, the contrast observed is only attributed to the passage of dynamics associated with the alpha-relaxation through our measurement window. Figure 3(f) shows the profile of the phase recorded at $42{ }^{\circ} \mathrm{C}$ across the interface between a PVAc island and the PS matrix [as shown in Fig. 3(c)]. We can estimate an upper limit of the spatial resolution (corresponding to half of the distance necessary to achieve the transition between the dielectric level of PVAc and PS) $\Delta \mathrm{x}$ around $\sim 40 \mathrm{~nm}$. This corresponds to the state-of-the-art resolution in EFM imaging when using standard probes, and it is also close to the theoretical resolution under our working conditions. ${ }^{16,17}$

In summary, we have developed a method that permits imaging the dynamics of nanostructured polymers with nanometer spatial resolution. This method of imaging dielectric losses is well decoupled from topography and can provide images with dielectric compositional contrast. For PS/PVAc polymer blends, strong phase separation was found. The glassy dynamics of the raised island-like domains was found to be nearly identical to that of pure PVAc films of similar thickness, whereas between these domains no dielectric response was found, consistent with pure PS behavior. Similar results were found on thicker films of PS/PVAc, though weak PVAc-like response was found in the PS regions indicative of possible buried PVAc domains. With further numerical modeling it may be possible to use this technique to analyze the three-dimensional structure of such films. The method described here makes it possible to study dielectric relaxation and molecular dynamics with nanometer scale spatial resolution in nanostructured polymers, nanocomposites, and other multicomponent soft materials, addressing questions of utmost importance such as the role of interfacial dynamics on the properties of such systems. Extending this method to ambient conditions would enable the study of soft hydrated materials such as proteins, and cell membranes, and investigate processes such as membrane potential formation, action potential propagation, or ion membrane transport. ${ }^{18}$ Work in this direction is in progress.

This work was supported by the Donostia Internacional Physics Center (DIPC), the Basque Country Government (Ref. No. IT-436-07, Depto. Educación, Universidades e Investigación), the Spanish Ministry of Science and Innovation (Grant No. MAT 2007-63681), European Community (SOFTCOMP program), and the National Science Foundation (Grant No. NSF DMR-0606090). C.R. and R.S. have equally contributed to the work.

${ }^{1}$ A. Serghei, M. Tress, and F. Kremer, Macromolecules 39, 9385 (2006).

${ }^{2}$ S. Napolitano, V. Lupascu, and M. Wübbenhorst, Macromolecules 41, 1061 (2008)

${ }^{3}$ R. Lund, L. Willner, A. Alegría, J. Colmenero, and D. Richter, Macromolecules 41, 511 (2008).

${ }^{4}$ L. Fumagalli, G. Ferrari, M. Sampietro, and G. Gomila, Nano Lett. 9, 1604 (2009).

${ }^{5}$ G. Gramse, I. Casuso, J. Toset, L. Fumagalli, and G. Gomila, Nanotechnology 20, 395702 (2009).

${ }^{6}$ C. Riedel, R. Arinero, P. Tordjeman, M. Ramonda, G. Lévêque, G. A. Schwartz, D. G. de Oteya, A. Alegría, and J. Colmenero, J. Appl. Phys 106, 024315 (2009)

${ }^{7}$ C. Riedel, R. Arinero, P. Tordjeman, G. Lévêque, G. A. Schwartz, A. Alegría, and J. Colmenero, Phys. Rev. E 81, 010801(R) (2010).

${ }^{8}$ P. S. Crider, M. R. Majewski, J. Zhang, H. Oukris, and N. E. Israeloff, Appl. Phys. Lett. 91, 013102 (2007).

${ }^{9}$ P. S. Crider, M. R. Majewski, J. Zhang, H. Oukris, and N. E. Israeloff, J. Chem. Phys. 128, 044908 (2008).

${ }^{10}$ G. A. Schwartz, E. Tellechea, J. Colmenero, and A. Alegría, J. Non-Cryst. Solids 351, 2616 (2005).

${ }^{11}$ G. A. Schwartz, J. Colmenero, and A. Alegría, J. Non-Cryst. Solids 353, 4298 (2007)

${ }^{12}$ M. Tyagi, A. Alegría, and J. Colmenero, J. Chem. Phys. 122, 244909 (2005).

${ }^{13}$ F. Kohlrausch, Pogg. Ann. Phys. 119, 352 (1863).

${ }^{14}$ G. Williams and D. C. Watts, Trans. Faraday Soc. 66, 80 (1970).

${ }^{15}$ I. Horcas, R. Fernandez, J. M. Gomez-Rodriguez, J. Colchero, J. GomezHerrero, and A. M. Baro, Rev. Sci. Instrum. 78, 013705 (2007).

${ }^{16} \mathrm{~S}$. Gómez-Moñivas, L. S. Froufe, R. Carminati, J. J. Greffet, and J. J. Saenz, Nanotechnology 12, 496 (2001).

${ }^{17}$ B. Bhushan and H. Fuchs, Applied Scanning Probe Methods II (Springer, New York, 2003), p. 312.

${ }^{18}$ J. Malmivuo and J. R. Plonsey, Biolectromagnetism: Principles and Applications of Bioelectric and Biomagnetic Field (Oxford University Press, New York, 1995). 